\title{
Physiological characteristics of rumen microbes and relation to
}

\section{diet and fermentation patterns}

\author{
By P. N. Hobson, Rowett Research Institute, Bucksburn, Aberdeen AB2 $9 S B$
}

Control of the rumen fermentation needs a basic knowledge of the rumen microbes. This short paper provides a few examples of work on the types and metabolic characteristics of the organisms and the influence of external conditions on their growth and metabolism.

The term 'fermentation patterns' in the rumen is generally understood to denote principally the proportions of three volatile fatty acids (VFA); acetic, propionic and butyric. The principal source of these fatty acids is carbohydrate fermentation. Lactate and succinate are also fermented, but the organisms responsible for fermentation of the latter are unknown. Deamination of amino acids usually appears to accompany carbohydrate fermentation, and Acidaminococcus fermentans, recently isolated by Elias (197I), is the first bacterium deriving energy from amino acid fermentations and unable to ferment carbohydrate known to occur as an appreciable proportion of a rumen flora. Control of carbohydrate fermentation will thus control the rumen fermentation pattern.

The growth of bacteria in media with excess of all growth factors except carbohydrate or nitrogen will be considered and it will be assumed that conditions are the same in the rumen but, for instance, inadequate minerals in the food could alter growth of the micro-organisms.

Carbohydrate fermentation is a conversion of sugars to VFA, lactic and succinic acids, ethanol, carbon dioxide and hydrogen. Some of these products may be further utilized by other bacteria with formation of VFA. The mixture of organisms producing these fermentation products consists of many types and one might ask whether it is possible to say that certain organisms are responsible for particular fermentation products? Identification of isolated bacteria with bacteria present in the rumen is often difficult because of pleomorphism. One can never be sure that all the main types have been isolated. Because of practical difficulties usually only a few strains of bacteria, grouped on the basis of a few tests, are selected for detailed study and determination of fermentation products. Fermentation products are usually complex and proportions of the different fermentation products often apparently vary widely between strains of a species, and which strain is predominant in the rumen may be difficult to tell. Different products may be formed by the same bacterium in fermenting different substrates, such as lactate or glucose, or even a polysaccharide or the monosaccharide derived from it. 
The $\mathrm{pH}$ at which bacteria grow can affect the fermentation products. With lacticacid bacteria, decreasing $\mathrm{pH}$ produces an increasing proportion of lactate in the products. Conversely, with Bacteroides amylophilus the proportion of VFA (formic and acetic) increased and succinic acid decreased as the culture $\mathrm{pH}$ was changed towards the minimum for growth at about $\mathrm{pH} 5.5$, but the reverse effect was suggested for Anaerovibrio lipolytica. The $\mathrm{pH}$ affects enzyme activities in cultures, both by the specific effect on activity and by an effect on production. For example, production of amylase by Bact. amylophilus was maximum at $\mathrm{pH}$ 6. Growth rate of organisms can have a profound effect on extracellular enzyme activities and also on intracellular enzymes as reflected by fermentation products. At low growth rates, Selenomonas ruminantium produced almost entirely acetic and propionic acids, whereas at high growth rates $50 \%$ of the products was lactic acid (Hobson, 1965; Hobson \& Summers, 1966, 1967; Henderson, Hobson \& Summers, 1969). Since these growth rates were, in some instances, controlled by carbohydrate concentration in the medium, the same effect is shown by batch cultures grown in different glucose concentrations; a high-glucose concentration gives high-lactate production (Hishinuma, Kanegasaki \& Takahashi, I968). Vries, Kapteijn, Beek \& Stouthamer (1970) showed a similar effect of growth rate on lactic-acid production by Lactobacillus casei. Gylswyck \& Roché ( 1970 ) found the proportion of lactic acid in products of fermentation of cellobiose by Butyrivibrio to be three times that in the products of cellulose fermentation-probably an effect of growth rates and soluble sugar concentrations. In the rumen this effect could cause the high concentration of fermentable sugar from easily degradable starch to set in train the symptoms of lactic acidosis, and an increased production of lactate could lead to higher propionate production. Another effect of carbohydrate concentration was shown in cultures of Bact. amylophilus, where the specific amylase activity under nitrogen-limited conditions was only $\mathrm{r} \cdot 5$, whereas under carbon limitation it was 20; protease activities were similarly affected. When nitrogen is low the bacterium diverts nitrogen from extracellular enzyme production to more essential activities (Henderson et al. ig69). Carbon or nitrogen limitation could similarly influence enzyme activities in the rumen.

All the factors so far mentioned influence the microbial cell yield from fermentation of a given amount of carbohydrate, in some instances by changing maintenance requirements for fermentation energy, or by producing uncoupled fermentations, Whether the fermentation is coupled to cell growth or uncoupled, fermentation acids of use to the animal are produced, but there is evidence from cultures of Bact. amylophilus that the proportions of products are different in coupled and uncoupled fermentations. Almost all information on the fermentation products of the protozoa has been obtained from washed-suspension experiments where fermentation occurs in the absence of cell growth. Adequate determination of the role of the protozoa in the rumen fermentation demands more work with growing cultures where the effects of growth conditions may be determined.

We have so far considered cultures with one fermentable substrate, but the rumen usually contains many fermentable substrates. As most rumen bacteria 
and protozoa can ferment several carbohydrates, the possibilities of the use of more than one substrate and the interactions of multiple substrates should be considered. Two substrates may be used at the same time by a culture of bacteria, but the presence of one substrate may suppress utilization of the other. Matales, Chian \& Silver $(1967)$ showed that in continuous culture with a mixed substrate a number of non-rumen bacteria utilized fructose and glucose together at low growth rates, but only glucose at high growth rates. Similar results were obtained with a mixed culture of sewage bacteria growing on lactose plus glucose. In the rumen, $S$. ruminantium is a lactate-utilizing bacterium, but whereas it utilized starch and lactate together (and here the fermentable sugar concentration would be low), in media containing glucose plus lactate, lactate utilization was suppressed (Hobson and Fina, unpublished). Hishinuma et al. (1968) found that, on glucose plus lactate, S. ruminantium preferentially utilized glucose in high concentration and lactate fermentation occurred, only after a lag period, when glucose was exhausted. At lowglucose concentrations, lactate fermentation occurred with no lag. This effect may have some bearing on lactate fermentation in the rumen. S. ruminantium ferments lactate by the succinate pathway, but high-carbohydrate concentrations in the diet would suppress lactate utilization by $S$. ruminantium and lactate might then be fermented by Peptostreptococcus elsdenii which ferments by the acrylate route, and whose relative growth rates on lactate and glucose (Hobson, Mann \& Oxford, 1958) suggest that lactate fermentation may not be so susceptible to catabolite repression by carbohydrates. Suppression of lactate fermentation in the rumen by carbohydrate would increase possibilities of lactic acidosis. Gylswyck \& Iabuschagne (I97I) found that ruminococci had almost the same growth rate on cellulose as cellobiose, whereas butyrivibrios grew much faster on cellobiose than cellulose. This type of result may explain the varying effects of easily fermentable carbohydrate on cellulose digestion. The predominant cellulolytic bacteria can vary and, if Butyrivibrio were predominant, the addition of easily fermented carbohydrate would result in growth on this rather than on cellulose. Also, such results emphasize that although a particular bacterium grows in vitro on a particular substrate it may not be of importance in the rumen.

Fermentation-product repression may change fermentation pathways. Propionate added to continuous cultures of Propionibacterium shermanii decreased the growth rate and rate of formation of propionate, but did not affect acetate production. Acetate addition affected acetate production, but it was required in higher concentrations than propionate (Jerusalimsky, i 967 ). Fermentation-product repression, if it occurs in the rumen, may serve to limit total production of an acid, and in the short term may help to stabilize acid production at some particular ratio of acids. Addition of an acid as part of a food might suppress formation of the acid and slow down the growth rate of the bacteria.

Summarizing, it might be said that it is difficult to predict from a few pure culture studies what fermentation products will be formed and what substrates will be utilized by bacteria in the rumen, and giving simultaneously different carbohydrates in a diet may give results different from those predicted from the 
fermentation in vitro and in vivo of single substrates. To keep fermentation patterns constant and the microbes working at full efficiency, the less the fluctuations in food concentrations the better.

Mixed cultures can introduce more variables. Many aspects of mixed culture growth and intermicrobial control have already been dealt with in detail (Hobson, r969, I97I); a few will be mentioned here. The rumen $\mathrm{pH}$ affects not only fermentation products, but growth rates of bacteria (Hobson, 1965). For instance, $S$. ruminantium had a maximum growth rate greater than a rumen Lactobacillus sp. at $\mathrm{pH} 6.7$, but the reverse was found at $\mathrm{pH} 6$ or below. In mixed cultures the selenomonad competed successfully with the Lactobacillus at high $\mathrm{pH}$, but at low $\mathrm{pH}$ the Lactobacillus was dominant (Hobson \& Stewart, I970). We have found that the minimum $\mathrm{pH}$ for growth of a number of representatives of the 'normal' rumen flora is about 6, but that Bact. amylophilus will grow down to about $\mathrm{pH} 5.5$, Pepto. elsdenii to 4.8 and the streptococci and lactobacilli even lower (Hobson, et al. 1958; Hobson, 1965; Hobson \& Summers, I967). A pH value of about 6 is unsuitable for rumen protozoa, with the possible exception of some Entodinium spp. These results show how some bacteria can take over in the rumen and why predominant rumen organisms vary with average rumen $\mathrm{pH}$. There are many interactions in the rumen culture, for instance in the provision of growth factors by one organism for another and, for example, Gylswyck (1970) found the addition of urea and branched-chain VFA (a growth factor for ruminococci) to a Teff-hay diet for sheep allowed the ruminococci to outgrow the Butyrivibrio and increase cellulolysis in the rumen.

Long-term fluctuations in the rumen flora are generally found and similar fluctuations have been noted in mixed cultures in vitro (Elias, Hobson and Summers, unpublished). It seems unlikely that mixed natural populations will be completely stable but, in the rumen, variations in VFA proportions may not follow microbial fluctuations, as compensating changes in micro-organisms may occur and several possible populations may produce similar fermentation products from the same, or different, foods. As an example of both aspects, the high-butyric acid in cattle fed on restricted-barley diets is ascribed to an exceptionally high protozoal population (Eadie, Hyldgaard-Jensen, Mann, Reid \& Whitelaw, 1970), but a high proportion of butyric acid is found in cattle fed on urea-fish meal-molasses, where the protozoal population is not high, the bacteria are entirely different and the butyric acid production is ascribed to bacterial fermentations (Elias, 197I).

From these considerations we can suggest how grinding, heating and other methods of food preparation will affect carbohydrate utilization and how food mixtures may be fermented, but can we add particular microbes to the rumen to digest food in a particular way? Experiments have shown that it is impossible to change an established flora by addition of a particular bacterium (Hobson \& Mann, 196r), but it is possible to build up a defined flora under gnotobiotic conditions, and this will behave predictably (Lysons, Alexander, Hobson, Mann \& Stewart, r97 I, and unpublished). Extensions of these experiments may show how a stable, more efficient, defined flora may be established under normal conditions, but it 
would already seem worth while to try adding missing organisms to the animals which do not respond naturally to food changes.

\section{REFERENCES}

Eadie, J. M., Hyldgaard-Jensen, J., Mann, S. O., Reid, R. S. \& Whitelaw, F. G. (I97o). Br. F. Nutr. 24, 157.

Elias, A. (1971). The rumen bacteria of animals fed on a high-molasses-urea diet. PhD Thesis, University of Aberdeen.

Gylswyck, N. O. van (1970). F. agric. Sci., Camb, 74, 169.

Gylswyck, N. O. van \& Labuschagne, J. P. L. (1971). F. gen. Microbiol. 66, 109.

Gylswyck, N. O. van \& Roché, C. E. G. (1970). F. gen. Microbiol. 64, i 1 .

Henderson, C., Hobson, P. N. \& Summers, R. (1969). In Continuous Culture of Microorganisms p. 189 [I. Málek, editor]. New York and London: Academic Press.

Hishinuma, F., Kanegasaki, S. \& 'Takahashi, H. (1968). Agric. biol. Chem. f. 32, 1325.

Hobson, P. N. (1965). F. gen. Microbiol. 38, I67.

Hobson, P. N. (I969). In Microbial Growth p. 43 [P. Meadow and S. J. Pirt, editors]. London: Cambridge University Press.

Hobson, P. N. (1971). Prog. ind. Microbiol. 9, 41.

Hobson, P. N. \& Mann, S. O. (196r). F. gen. Microbiol. 24, i. (Title only.)

Hobson, P. N., Mann, S. O. \& Oxford, A. E. (1958). Y. gen. Microbiol. r9, 462.

Hobson, P. N. \& Stewart, C. S. (1970). F. gen. Microbiol. 63, xi.

Hobson, P. N. \& Summers, R. (1966). Nature, Lond. 209, 736.

Hobson, P. N. \& Summers, R. (1967). F. gen. Microbiol. 47, 53.

Jerusalimsky, N. D. (1967). In Microbial Physiology and Continuous Culture p. 23 [E. O. Powell, editor]. London: HM Stationery Office.

Lysons, R. J., Alexander, 'T., Hobson, P. N., Mann, S. O. \& Stewart, C. S. (1971). Res. vet. Sci. $\mathbf{1 2}, 486$.

Matales, R. I., Chian, S. K. \& Silver, R. (I967). In Microbial Physiology and Continuous Culture p. 233 [E, O. Powell, editor]. London: HM Stationery Office.

Vries, W. de, Kapteijn, W. M. C., Beek, E. G. van der \& Stouthamer, A. H. (1970). Y. gen. Microbiol. 63,333 . 